\section{The photoreceptor}

This issue of Eye contains the papers from the 1997 Cambridge Symposium, which brought together basic scientists and clinicians who have an interest in photoreceptor structure, physiology and disease. The Cambridge Symposium last addressed the subject of the outer retina in 1983, and in the intervening 14 years there have been tremendous advances made in our understanding of the biology of the retina and the mechanisms underlying retinal disease.

Diseases of the outer retina are an important cause of childhood and adult blindness; most are due to genetic mutations and are at present untreatable. Advances in molecular biology have enabled the specific genetic mutations to be identified in many of these disorders and Inglehearn in this issue has summarised the main advances in this area. It is perhaps not surprising that mutations of many of the genes encoding proteins involved in

phototransduction are associated with photoreceptor degeneration, but other mutations causing outer retinal dystrophies encode proteins of uncertain function and the finding of such genes has opened up new areas of research into the biochemistry of the retina. They have also served to highlight how much more we need to understand about normal retinal function.

Although the specific mutations causing disease may be identified, the mechanism by which this leads to photoreceptor dysfunction and cell death is still ill understood. Rhodopsin is the phototransduction protein most commonly associated with retinal dystrophy and over 100 different mutations have been described to date. Most cause autosomal dominant retinitis pigmentosa, although specific mutations may be associated with autosomal recessive retinitis pigmentosa and congenital stationary night blindness. There is considerable phenotypic variation seen even with disease associated with mutations of the rhodopsin gene and such variation cannot be understood without a clear understanding of the normal structure and function of the protein. Schertler in this issue discusses current knowledge of the three-

\section{A.T. MOORE}

dimensional structure of rhodopsin and Deretic reviews the trafficking of rhodopsin within the rod photoreceptor. The other transduction proteins have been less fully investigated, but study of the functional consequences of specific mutations has also been aided by the development of transgenic models and such models may also be helpful in improving our understanding of the normal transduction process (see Baylor and Burns in this issue).

Naturally occurring animal models of inherited retinal dystrophies also exist and are summarised in the paper by Petersen-Jones. These models are an important resource both for the study of disease mechanisms and for testing potential new forms of treatment such as gene therapy, the use of growth factors to stabilise retinal function and retinal or retinal pigment epithelial transplantation. Careful scientific studies of the effects of new forms of therapy in animal models (such as those described by Lund et al. in this issue) are essential before clinical trials can begin in man. It is also important that we have a good understanding of the natural history of retinal dystrophies and have the ability to detect changes in retinal function that may follow the introduction of treatment strategies. This has been helped by the development of new methods of imaging the photoreceptor layer and more detailed methods of electrophysiological and psychophysical testing of scotopic and photopic function.

It is not possible in a short editorial to do justice to the many other topics that were discussed at this symposium. I am sure the papers in this issue will stimulate interest and serve to highlight the importance of basic science in understanding disease processes in the retina. The challenge for both scientists and clinicians remains to harness the tremendous advances in molecular biology, cell biology, retinal physiology and other scientific disciplines to identify effective treatment strategies for this group of blinding disorders. This will necessitate a multidisciplinary approach with close collaboration between scientists and clinicians.
A.T. Moore Consultant Ophthalmic Surgeon

Addenbrooke's Hospital Hills Road

Cambridge CB2 2QQ, UK 